\title{
Coexistence of superconductivity and ferromagnetism in
}

\section{cluster-assembled Sn-Co nanocomposites}

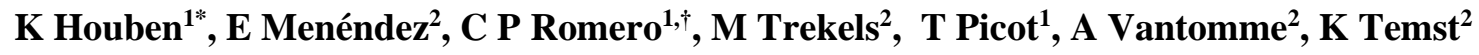 \\ and M J Van Bael ${ }^{1}$
}

${ }^{1}$ KU Leuven, Laboratory of Solid-State Physics and Magnetism, Celestijnenlaan 200 D, 3001 Leuven, Belgium

${ }^{2} \mathrm{KU}$ Leuven, Instituut voor Kern- en Stralingsfysica, Celestijnenlaan 200 D, 3001 Leuven, Belgium

E-mail: kelly.houben@fys.kuleuven.be

\begin{abstract}
The coexistence of superconductivity and ferromagnetism is investigated in granular Sn-Co nanocomposites. The nanocomposites have been prepared by co-deposition of Sn atoms and Co clusters, the morphology and composition of which can be tuned by varying the deposition rate of Co clusters relative to $\mathrm{Sn}$ atoms. Flat isolated $\mathrm{Sn}$ islands are obtained at zero or low Co cluster flux, while granular nanocomposites are formed with increasing Co cluster flux, reaching Co concentrations up to 44 vol.\%. Interfaces with a low electronic transparency between superconductor and ferromagnet are obtained by a combination of the granular nature of the nanocomposites and the formation of Sn-Co alloys at the Sn/Co interfaces. The structure and composition of the nanocomposites have been thoroughly characterized by atomic force microscopy, x-ray diffraction and conversion electron Mössbauer spectroscopy. Over the entire Co concentration range, the hybrids show a ferromagnetic response. The superconducting phase boundary and the Meissner response depend on the morphology and composition of the nanocomposites. In particular, the superconducting critical temperature decreases with increasing Co concentration, while the Meissner response varies from a reversible to a strongly

\footnotetext{
* Corresponding author

† Present address: Departamento de Física, Universidad Técnica Santa Maria, Av. España 1680, Valparaiso, Chile
} 
hysteretic behaviour depending on the morphology of the samples with different Co content. The persistence of superconductivity at high Co concentrations is attributed to a suppression of the superconducting proximity effect in these nanocomposites, which is ascribed to the low interface transparency between the $\mathrm{Sn}$ and Co components that make up these hybrid systems.

\section{Introduction}

Coexistence between superconductivity and ferromagnetism is counter-intuitive. When applying a magnetic field to a superconductor, the spins of the electrons in a Cooper pair tend to align along the magnetic field resulting in the destruction of superconducting behaviour (the magnetic pair breaking effect). Moreover, in artificially created superconductor/ferromagnet (S/F) hybrid systems in which the magnetic component is a normal conductor, Cooper pairs and normal electrons can cross the S/F interface (the so-called superconducting proximity effect) which causes a further suppression of superconductivity [1, and references therein]. Despite these two pair breaking mechanisms, there is a rich variety of interesting phenomena that can occur when combining a ferromagnet with a superconductor, e.g. S/F proximity effects [1], vortex pinning [2-4], fluxoid quantization [5], magnetic-field-induced superconductivity [6], enhanced surface superconductivity [7], domain wall superconductivity [8]. In most of the studied S/F hybrid systems both the magnetic pair breaking effect and the superconducting proximity effect occur, leading to a strong interaction between $\mathrm{S}$ and $\mathrm{F}$ and a fast decrease in the superconducting transition temperature with increasing F concentration [9 - 16]. In many cases, a good interface transparency is desired in order to study the intimate mutual interactions between the $\mathrm{S}$ and $\mathrm{F}$ constituents governed via proximity coupling [15, 17 - 19]. In this work the focus is on hybrid systems with a low $\mathrm{S} / \mathrm{F}$ interface transparency, i.e. where the proximity effect is suppressed. Such systems are also of particular interest when studying and modulating the S/F interaction solely due to the magnetic pair breaking effect [6].

Not only the hybrid composition, but also the morphology strongly influences physical properties such as superconductivity and magnetism [20, 21]. In the research field of S/F hybrids, both highly ordered (layered or patterned) systems $[2,5-7,17-19,22,23]$ as well as systems with random distributions of $\mathrm{F}$ atoms in a $\mathrm{S}$ matrix [9 - 14] have been studied theoretically and experimentally. In the latter 
systems, typical concentrations of $\mathrm{F}$ atoms range from less than 1 at. $\%$ to a few at.\%, and a

considerable decrease in $T_{C}$ (up to 35\%) is observed for relatively low F concentration (7 at.\%).

An intermediate case between these extremes, both regarding ordering and modulation length scale, are the nanocomposites with a nanoscale modulation of $\mathrm{S}$ and $\mathrm{F}$ on length scales comparable to or even considerably smaller than the penetration depth or the superconducting coherence length. Interesting phenomena have been reported in such nanocomposite S/F systems, e.g. glassy vortex dynamics [24], spontaneous vortex phases $[15,16]$, new pinning mechanisms $[25], \ldots$

In this work, we investigate hybrid $\mathrm{S} / \mathrm{F}$ systems in this intermediate range with a granular morphology on length scales comparable to the superconducting coherence length and in which low transparency interfaces are intentionally created at the nanoscale. The nanogranular morphology as well as the composition of the S/F nanocomposites is manipulated by using preformed nanometer-sized Co clusters as building blocks and by tuning the deposition rate of the Co cluster beam during codeposition with a Sn atomic beam. The mobility of these two subsystems is very different and the deposition rate of Co clusters will further affect the effective mobility of the $\mathrm{Sn}$ atoms. The resulting granular morphology as well as the partial mixing of Sn and Co at the Sn-Co interfaces result in a low electronic interface transparency between superconducting and magnetic constituents. We investigate how morphology and composition influence the superconducting behaviour and the coexistence between superconductivity and ferromagnetism in systems in which the proximity effect is reduced. It is found that the low transparency of the $\mathrm{S} / \mathrm{F}$ interfaces enables the $\mathrm{S} / \mathrm{F}$ coexistence to persist up to high concentrations of the ferromagnetic component. Furthermore, the Meissner response is strongly modulated by the morphology and composition of the nanocomposites.

We have selected nanocomposite systems composed of Sn and Co constituents. Sn can exist in two phases: $\alpha$ - and $\beta$-Sn (white tin). The $\beta$-Sn phase, stable at room temperature, has a body-centered tetragonal (BCT) structure and is the only phase showing superconductivity (bulk critical temperature of $3.72 \mathrm{~K}[26])$. Furthermore, the ${ }^{119} \mathrm{Sn}$ isotope allows for structural characterization by Mössbauer spectroscopy. Tin is known to form alloys with a variety of elements, among them Co. Thus, when producing Sn-Co hybrid systems, the formation of Sn-Co compounds at the interface is expected. Co concentrations of $0,14,24$ and 44 vol.\% were uniformly chosen within the range reported in 
literature from a few atomic percent for paramagnetic doping of superconductors [9 - 14] up to several tens of percent when larger amounts of magnetic nanoparticles are incorporated in a superconducting matrix $[15,16,25]$.

\section{Experimental details}

Co clusters were produced using an ultrahigh vacuum (UHV) laser-vaporization cluster source. A detailed description of this cluster source can be found elsewhere [27]. Two pulsed Nd:YAG lasers (wavelength $=532 \mathrm{~nm}$, repetition rate $=10 \mathrm{~Hz}$ ) are used to ablate material from a high purity Co target. As the lasers are triggered, a synchronized pulse of helium $(\mathrm{He})$ gas enters the source chamber to cool down the plume of material created at the surface of the target and cluster nucleation starts.

This mixture of gas, atoms and clusters expands into the vacuum through a conical nozzle after which cluster growth stops. By differential pumping, a well-collimated cluster beam is formed. The Co clusters have a size distribution centered at a diameter of about $2 \mathrm{~nm}$ with a full width at half maximum of $0.6 \mathrm{~nm}$. The clusters are deposited in a low energy regime $(<1 \mathrm{eV} /$ atom $)$. This ensures a soft landing, thus minimizing cluster deformation and damage to the substrate. The base and working pressures in the deposition chamber are $1 \times 10^{-9} \mathrm{mbar}$ and $2 \times 10^{-7} \mathrm{mbar}$, respectively. These vacuum conditions minimize contamination of the deposited clusters. The deposition chamber is equipped with a Knudsen-cell from which $\mathrm{Sn}$ is evaporated. During deposition, the $\mathrm{Si} / \mathrm{SiO}_{2}$-substrates were cooled down to $190 \mathrm{~K}$ using liquid nitrogen. The cluster flux is monitored with a calibrated quartz microbalance. By varying the Co cluster flux, the Co concentration in the S/F sample can be tuned. Different Sn-Co hybrid systems with Co volume concentrations ranging between 0 and $44 \%$ have been prepared.

To study the sample topography, the films were transported in air to an atomic force microscope (AFM) (Digital Instruments Dimension system). Further structural characterization was performed by XRD and grazing incidence X-ray diffraction (GIXRD) with an incident angle of $5^{\circ}$ (PANalytical X'pert PRO X-ray diffractometer using $\mathrm{Cu} \mathrm{K}_{\alpha}$ radiation). While XRD probes the planes parallel to the sample surface only (i.e., out-of-plane characterization), GIXRD gives information about planes which 
are tilted with respect to the sample surface. To obtain the crystallite size (average coherently diffracting crystallite size) and the lattice parameters of the different phases, Rietveld refinement [28] of the XRD and GIXRD patterns was performed using the MAUD software [29]. Additional information on the composition of the samples was obtained by CEMS measurements [30] using the ${ }^{119} \mathrm{Sn}$ Mössbauer isotope. $\mathrm{A}^{119 \mathrm{~m}} \mathrm{Sn}$ source in a $\mathrm{CaSnO}_{3}$ matrix is used, with a nominal activity of 10 mCi. The conversion electrons are detected after being emitted during de-excitation of the resonantly excited ${ }^{119} \mathrm{Sn}$ nucleus. The Mössbauer spectra were least square fitted with the program Recoil [31] using a Lorentzian line shape. All values of isomer shifts and quadrupole splittings are given with respect to $\mathrm{CaSnO}_{3}$ [32]. All spectra were recorded at room temperature. The superconducting and magnetic behaviour of the Sn-Co hybrid systems were investigated by SQUID magnetometry (Quantum Design, MPMS-XL).

\section{Results and discussion}

\subsection{Structural characterization}

A pure $\mathrm{Sn}$ film with a nominal thickness of $60 \mathrm{~nm}$ is investigated, along with three hybrid systems having the same nominal Sn thickness with different Co concentrations (ranging from 14 up to 44 vol. $\%)$. The change in morphology by adding Co clusters to the Sn system is investigated by AFM. In figure 1, AFM images of Sn-Co systems with four different Co concentrations $(0,14,24$ and 44\%) are shown.
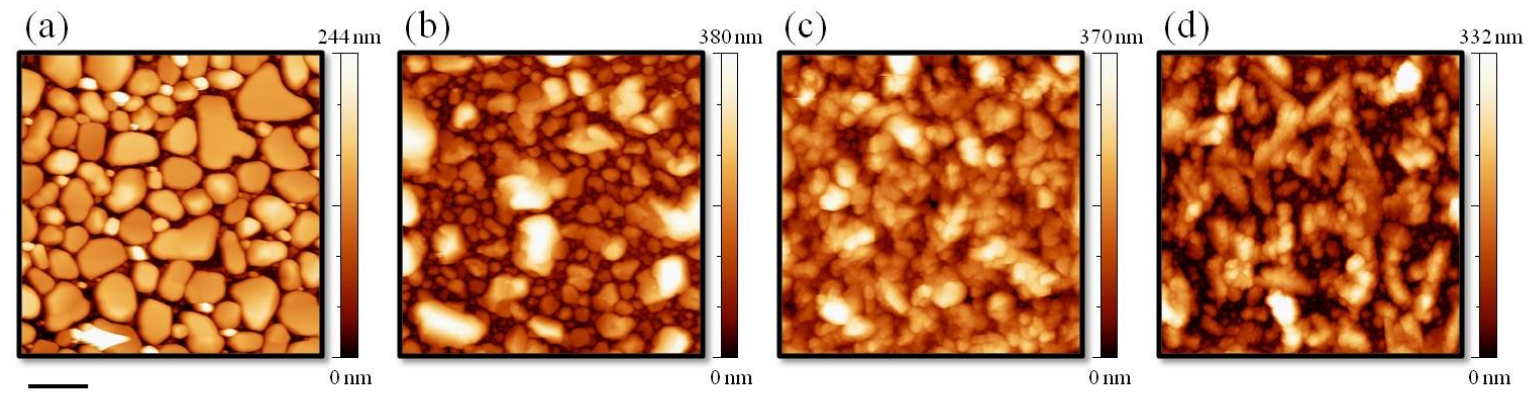

$\overline{1 \mu \mathrm{m}}$

Figure 1. AFM images $\left(5 \times 5 \mu \mathrm{m}^{2}\right)$ of Sn-Co hybrid systems with increasing Co concentrations: (a) pure Sn film, (b) Sn film with $14 \%$ Co, (c) Sn film with $24 \%$ Co, and (d) Sn film with $44 \%$ Co.

For the pure Sn film, a root-mean-square (rms) roughness of $58 \pm 3 \mathrm{~nm}$ is found for a $5 \times 5 \mu \mathrm{m}^{2}$ area, 
while a rms roughness of $2 \pm 2 \mathrm{~nm}$ is found for $400 \times 400 \mathrm{~nm}^{2}$ areas within the islands. Adding Co clusters to the $\mathrm{Sn}$ film initially increases the rms roughness for a $5 \times 5 \mu \mathrm{m}^{2}$ area to $86 \pm 4 \mathrm{~nm}$. When comparing the hybrid Sn-Co systems, a decrease in rms roughness from $86 \pm 4 \mathrm{~nm}$ down to $46 \pm 2 \mathrm{~nm}$ is observed with increasing Co concentration from $14 \%$ up to $44 \%$ Co. By adding Co clusters, the morphology changes from island-like for the pure Sn film to a granular-like structure. Due to the low kinetic energy deposition regime $(<1 \mathrm{eV} /$ atom $)$, the Co clusters are hardly deformed upon impact with the $\mathrm{SiO}_{2}$ surface [33]. Moreover, from the random distribution of deposited Co clusters on $\mathrm{SiO}_{2}$ surface (see supplementary information) it can be concluded that the diffusive mobility of the deposited clusters remains low [34].

To produce systems with different Co concentrations, the Co cluster flux was adjusted between 0.02 $\AA /$ s for the system with $14 \%$ Co to $0.06 \AA /$ s for the $44 \%$ Co sample. The Sn atom flux was kept constant at $0.17 \AA /$ s for all samples. Based on the topography observed by AFM, a growth model for $\mathrm{Sn}$ is proposed, and schematically presented in figure 2 . The resulting morphology of this growth model is also consistent with the results of XRD, CEMS and SQUID measurements, which will be presented further in this paper.

The island-like growth morphology of pure $\mathrm{Sn}$ is a result of the pronounced diffusive mobility of $\mathrm{Sn}$ atoms on a $\mathrm{SiO}_{2}$ substrate and the minimization of the overall surface and interface energy [35]. At room temperature, $\mathrm{Sn}$ atoms can migrate rapidly over a $\mathrm{SiO}_{2}$ substrate [36]. From the observed topography in figure 1 (a), it can be concluded that $\mathrm{Sn}$ atoms can migrate on a $\mathrm{SiO}_{2}$ substrate even at temperatures below room temperature (i.e., $190 \mathrm{~K}$ ). A schematic representation of a $\mathrm{Sn}$ film is shown in figure 2 (a).

The observed topography evolution with increasing Co concentration, figure 1 (b)-(d), can be explained by the dissimilar mobility of Co clusters compared to that of the deposited Sn atoms. When Co clusters are co-deposited, they inhibit the diffusion of Sn atoms and hamper the island formation. At low Co cluster flux, $\mathrm{Sn}$ atoms impinging on the $\mathrm{SiO}_{2}$ substrate have time to coalesce into small $\mathrm{Sn}$ islands before they encounter an immobile Co cluster. This way, smaller isolated Sn islands are formed, separated by different SnCo compounds, as is schematically represented in figure 2 (b). When the Co cluster flux is increased, as for the $24 \%$ Co sample, the Sn atoms on the substrate do not 
have enough time to form small islands before encountering a Co cluster on the substrate. Instead, a

granular system is formed, composed of $\mathrm{Co}, \mathrm{Sn}, \mathrm{SnCo}$ and $\mathrm{Sn}_{2} \mathrm{Co}$ components, as proposed in figure 2 (c).

If the Co cluster flux is even further increased, as for the $44 \%$ Co system, the $\mathrm{Sn}$ atoms can only form very small $\mathrm{Sn}$ areas separated by $\mathrm{Co}, \mathrm{SnCo}$ and $\mathrm{Sn}_{2} \mathrm{Co}$ components and no percolating path for the superconducting current is formed between these Sn areas. A schematic representation is suggested in figure $2(d)$.

(a)

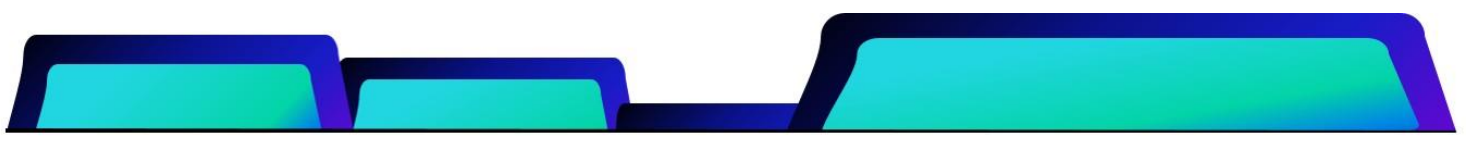

(b)

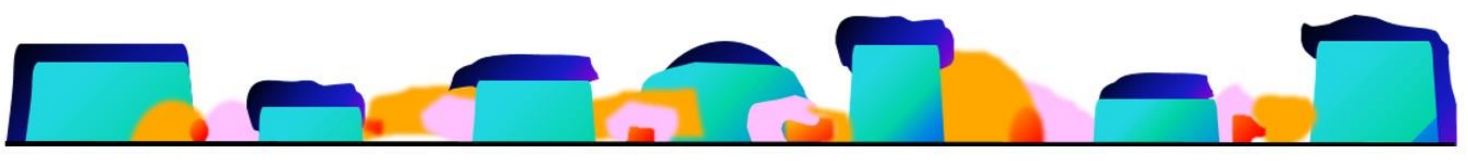

(c)

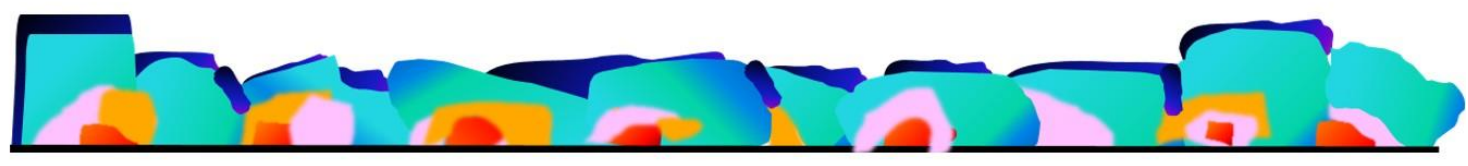

(d)
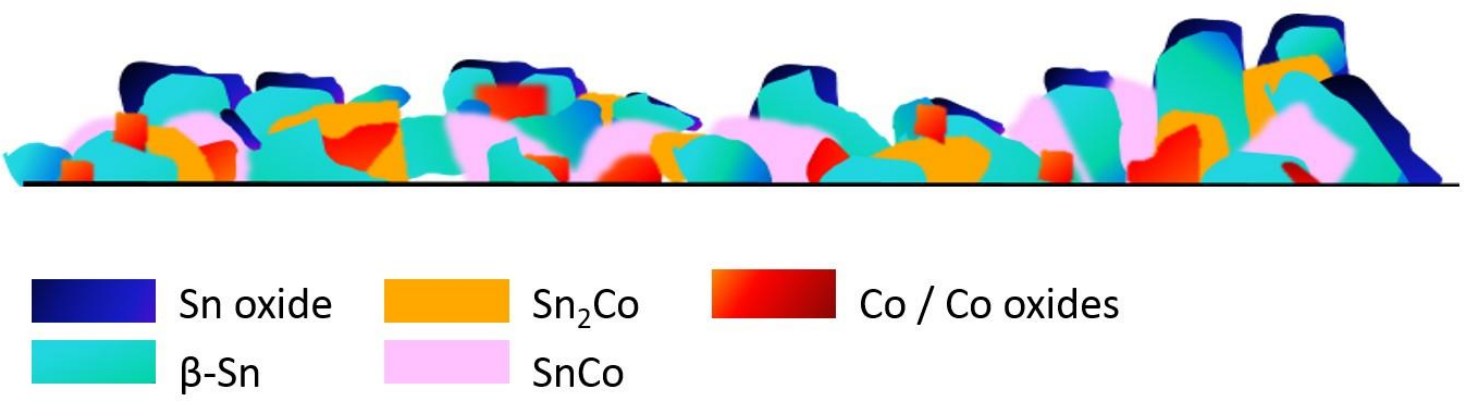

Figure 2. Schematic representation of the suggested morphology of Sn-Co hybrids with increasing Co concentration; (a): side view of a pure Sn film, Sn forming large islands; (b) side view of a Sn-Co hybrid system with $14 \%$ Co, showing that Sn forms smaller islands in comparison to the pure Sn film, which are separated from each other; (c): side view of a Sn-Co hybrid system with $24 \%$ Co, showing that $\mathrm{Sn}$ forms small areas forming a percolating path; and (d) side view of a Sn-Co hybrid system with $44 \%$ Co, proposing that $\mathrm{Sn}$ gets divided up into very small areas.

XRD measurements have been performed to determine the $\beta$-Sn crystallite size for the Sn-Co hybrids with different Co concentrations, and to identify the different phases. The XRD pattern of the pure Sn film is consistent with a highly textured $\beta$-Sn phase oriented along (200) planes (not shown). Even 
though this preferential alignment is also present in the $\beta$-Sn phase of the nanocomposite samples, traces of (101) XRD peaks are also observable. This suggests that co-deposition results in a $\beta$-Sn phase with a higher degree of polycrystallinity.

The GIXRD patterns of the Sn-Co systems with different Co content as well as the GIXRD pattern of a reference pure Sn film are shown in figure 3.

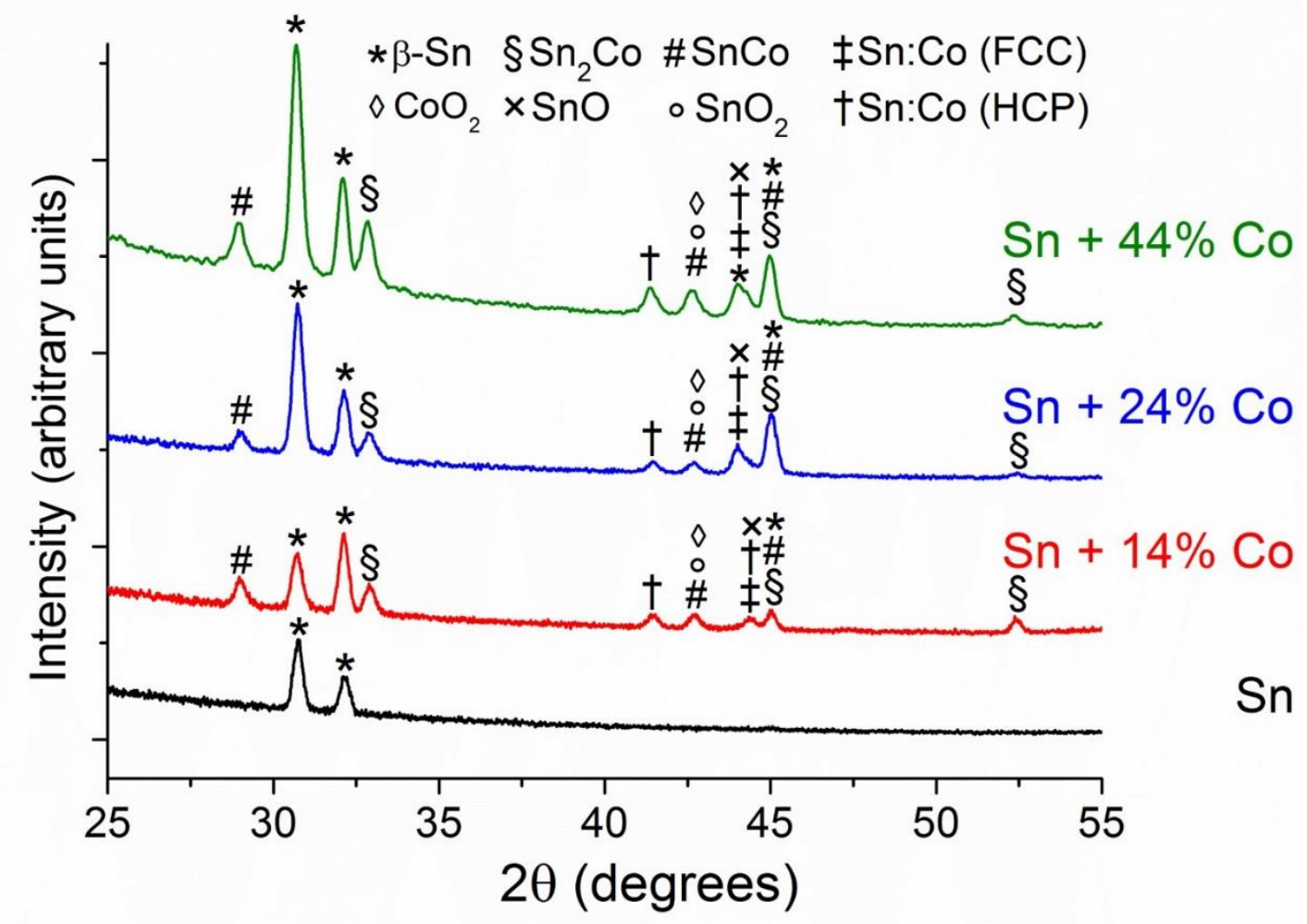

Figure 3. GIXRD patterns for Sn-Co hybrid systems with different Co concentrations. Symbols indicate the identification of the peaks appearing in the GIXRD patterns. The crystalline planes corresponding to the different peaks occurring in the GIXRD patterns are listed in table 1A in the supplementary information. Co-rich solid solutions are indicated by Sn:Co.

In figure 3 , the peaks marked with $\ddagger$ and $\dagger$ correspond to a Co-rich solid solution (Co with up to 5\%

Sn) face-centered cubic (FCC) [37] and a Co-rich solid solution (Co with $5-10 \%$ Sn) hexagonal close-packed (HCP) [38], respectively. The presence of $\beta$-Sn XRD peaks confirms the polycrystalline nature of the $\beta$-Sn formed in all samples. However, the nanocomposite systems show more $\beta$-Sn peaks (peaks located at 44 and 45 degrees are only present in the nanocomposite samples) than the reference sample (i.e., pure $\beta-\mathrm{Sn}$ ), indicating the higher degree of polycrystallinity of the $\beta$-Sn phase in the nanocomposite samples. The GIXRD patterns of the Sn-Co systems exhibit additional peaks, which 
can be identified as $\mathrm{SnCo}$ and $\mathrm{Sn}_{2} \mathrm{Co}$ compounds. The crystalline planes corresponding to the different peaks occurring in the GIXRD patterns shown in figure 3 are listed in table $1 \mathrm{~A}$ in the supplementary information.

Table 1 shows the average crystallite size of the $\beta$-Sn phase for the different Sn-Co hybrid systems, as obtained from Rietveld refinement of the GIXRD measurements. The decreasing trend in the crystallite size of the $\beta$-Sn phase with increasing Co concentration is consistent with the observed topography in the AFM images shown in figure 1 and the morphology of the Sn-Co hybrids as schematically proposed in figure 2 .

Table 1. Crystallite size of the $\beta$-Sn phase obtained from Rietveld refinement of the GIXRD data.

\begin{tabular}{cc}
\hline Sample & $\begin{array}{c}\text { Crystallite size of } \\
\beta-S n(n m)\end{array}$ \\
\hline Sn & $141 \pm 3$ \\
Sn $+14 \%$ Co & $91 \pm 5$ \\
Sn $+24 \%$ Co & $98 \pm 4$ \\
Sn $+44 \%$ Co & $71 \pm 4$ \\
\hline
\end{tabular}

Figure 4 shows the results of CEMS measurements carried out on the four samples. Mössbauer spectroscopy is a very powerful technique for obtaining information on the oxidation state and composition for all compounds containing the Mössbauer isotope $\left({ }^{119} \mathrm{Sn}\right.$ in this case) [39]. 


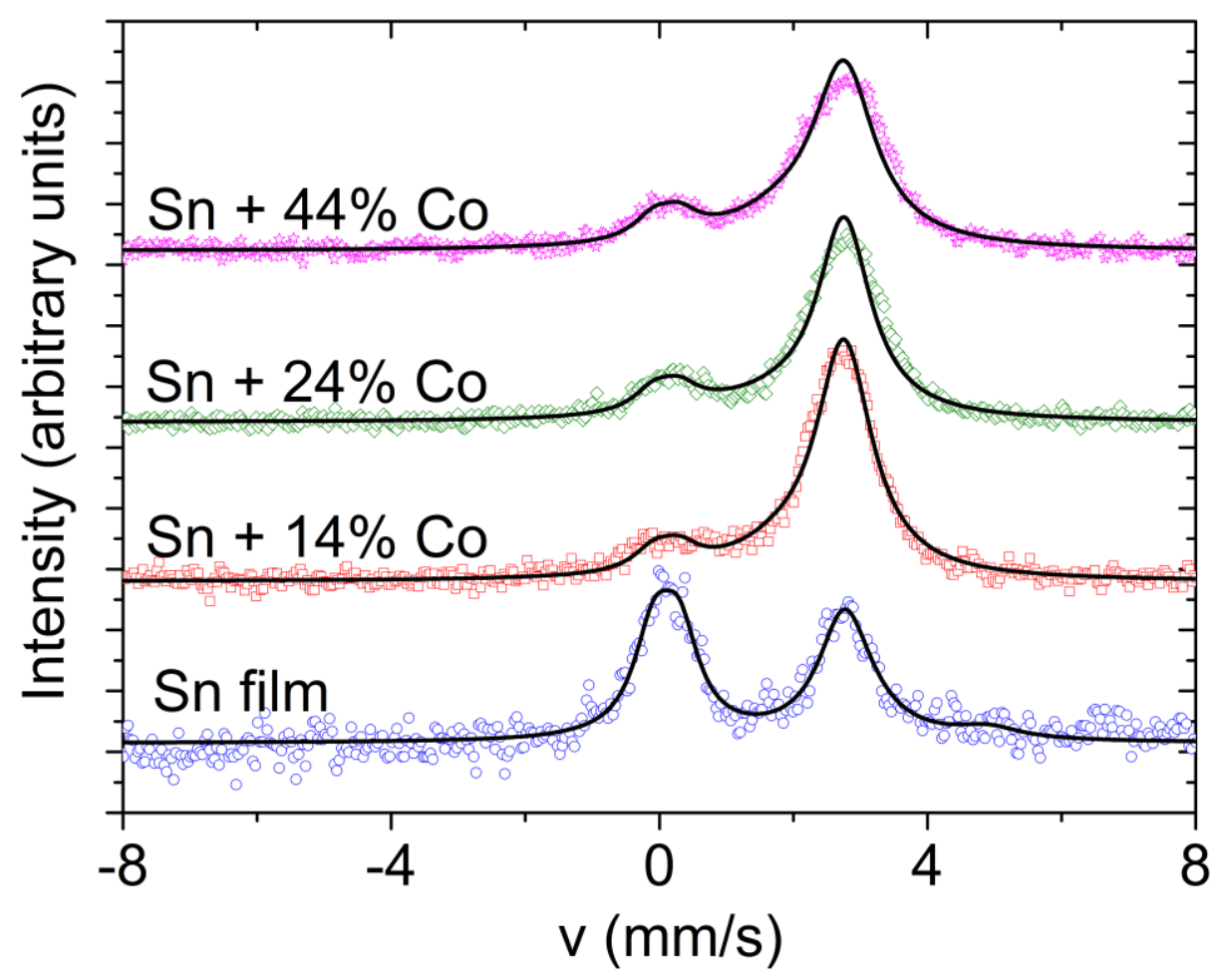

Figure 4. CEMS measurements of a Sn film (open circles) and Sn-Co hybrid systems with different Co concentration ( $14 \%$ Co: open squares; $24 \%$ Co: open diamonds and $44 \%$ Co: open stars). The fit of the CEMS data is shown with a solid line.

The CEMS data for the pure Sn film can be fitted by a singlet site and two doublet sites. From the fit we find that the singlet site has an isomer shift $(\delta)$ of $2.76 \mathrm{~mm} / \mathrm{s}$, which corresponds to pure $\mathrm{Sn}$. The two doublet sites have an isomer shift of $3.9 \mathrm{~mm} / \mathrm{s}$ and $0.10 \mathrm{~mm} / \mathrm{s}$ and quadrupole splittings $(\Delta)$ of $1.84 \mathrm{~mm} / \mathrm{s}$ and $0.38 \mathrm{~mm} / \mathrm{s}$, which correspond to $\mathrm{SnO}$ and $\mathrm{SnO}_{2}$, respectively.

The CEMS data for the different Sn-Co hybrid systems can be fitted by a Sn singlet site and by three doublet sites for $\mathrm{SnO}_{2}$, SnCo and $\mathrm{Sn}_{2} \mathrm{Co}$. The two doublet sites for $\mathrm{SnCo}$ and $\mathrm{Sn}_{2} \mathrm{Co}$ correspond to isomer shifts of $1.8 \mathrm{~mm} / \mathrm{s}$ and $2.14 \mathrm{~mm} / \mathrm{s}$ and quadrupole splittings of $1.6 \mathrm{~mm} / \mathrm{s}$ and $0.78 \mathrm{~mm} / \mathrm{s}$, respectively [40, 41]. Both, $\mathrm{Sn}_{2} \mathrm{Co}$ and SnCo could also be identified in the GIXRD measurements (see figure 3). Aside from the isomer shift and quadrupole splitting, also the relative amounts of the different spectrum components can be extracted from the fits of the CEMS measurements, see figure 5, which provide a link between the spectrum components and the composition of the Sn-containing constituents. 


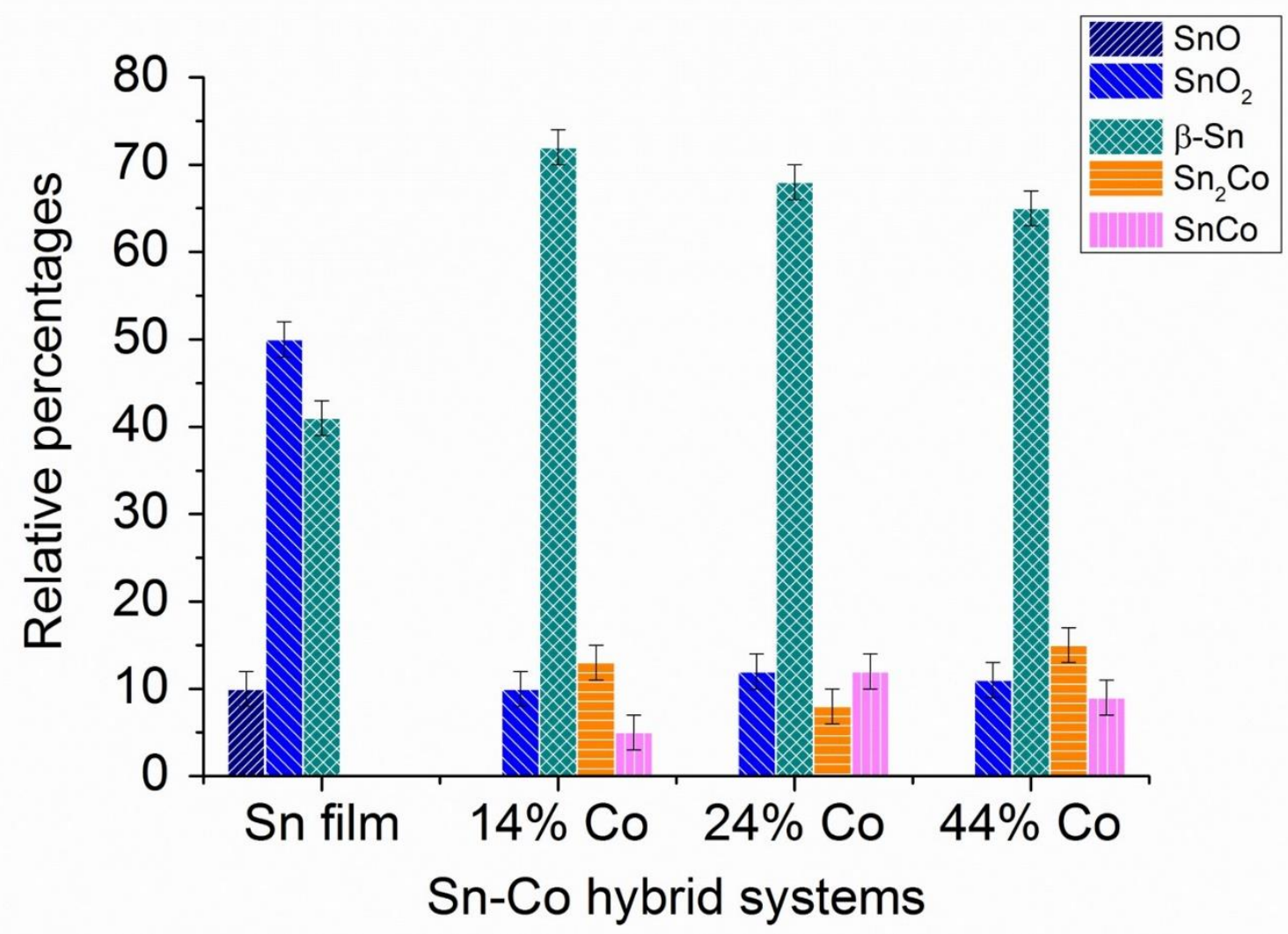

Figure 5. Relative percentages of the different spectrum components for all Sn-Co hybrid systems, obtained from fitting the CEMS data.

Upon adding Co to the Sn films, the doublet site corresponding to SnO disappears completely from the CEMS spectrum, the $\mathrm{SnO}_{2}$ amount decreases, and the $\mathrm{Sn}$ amount increases. The possible presence of Co-oxides in the systems can not be excluded, but can not be studied via CEMS.

Upon further addition of $\mathrm{Co}$, the amounts of $\mathrm{SnO}_{2}$ and $\mathrm{Sn}$ remain approximately constant within experimental accuracy. A slightly decreasing trend of the pure $\mathrm{Sn}$ fraction could be discerned with increasing Co concentration. The amounts of $\mathrm{Sn}_{2} \mathrm{Co}$ and $\mathrm{SnCo}$ remain approximately constant with increasing Co amount, with only a slight increase of the total amount of the Sn-Co compound as the Co concentration grows.

We recall that CEMS only probes the different chemical environments of Sn. When the Co concentration is increased from 14 to $44 \%$, the main fraction of additional Co is therefore expected not to form compounds with $\mathrm{Sn}$ (there will be some doping of Co with $\mathrm{Sn}$ atoms giving rise to a Co solid solution as evidenced in the GIXRD characterization). Due to the lack of knowledge about the Lamb- 
Mössbauer factor for $\mathrm{Sn}_{2} \mathrm{Co}$, no absolute percentages can be obtained for the different $\mathrm{Sn}$-Co hybrid systems.

\subsection{Magnetic and superconducting properties}

SQUID magnetization measurements have been performed to probe the magnetic behaviour of the $\mathrm{Sn}$ -

Co hybrid systems and to probe the extent of coexistence of superconductivity and ferromagnetism.

The external magnetic field $H_{\text {applied }}$ was applied perpendicular to the sample plane for all measurements (i.e. out-of-plane configuration). The conversion factor from SI units to Gaussian units is provided in the supplementary information. Figure 6 shows the room temperature $M\left(H_{\text {applied }}\right)$ curves demonstrating the ferromagnetic behaviour of the Co-containing hybrids (Co concentration ranging from $14 \%$ up to $44 \%$ Co). The Sn-Co systems do not show significant magnetic anisotropy when comparing in-plane vs. out-of-plane measurements, indicating a rather isotropic shape of the ferromagnetic parts. The coercive field decreases from $12 \mathrm{mT}$ to $4 \mathrm{mT}$ with increasing Co concentration. For larger Co concentrations, increased percolation between ferromagnetic parts is expected [42]. It was found that $\mathrm{Sn}_{1-\mathrm{x}} \mathrm{Co}_{\mathrm{x}}$ with $x$ down to 0.49 still exhibits ferromagnetic properties [43]. This implies that the SnCo compound which is present in our Sn-Co hybrid systems (as deduced from XRD and CEMS measurements) will also contribute to the ferromagnetic signal, together with the Co-rich solid solutions.
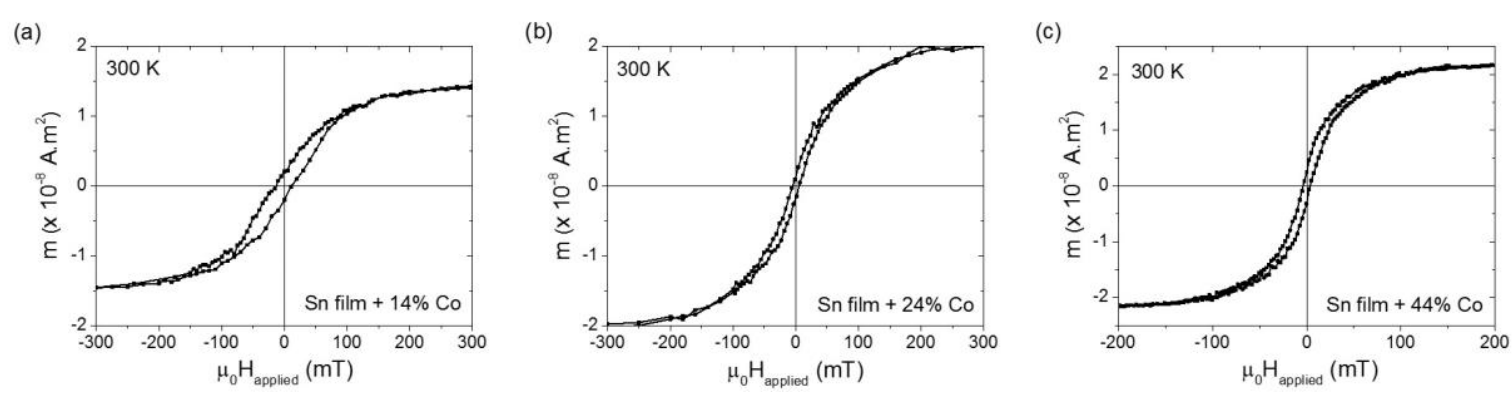

Figure 6. Magnetic moment as a function of the applied magnetic field at room temperature for Sn-Co systems with different Co concentrations; (a): Sn film with 14\% Co; (b): Sn film with $24 \%$ Co; (c): Sn film with $44 \%$ Co.

For the pure Sn film, no ferromagnetic response is expected at room temperature. After subtracting the linear diamagnetic signal, a small but unavoidable non-intrinsic magnetic background of the order of $10^{-9} \mathrm{Am}^{2}$ will however always remain, even for pure Sn films (see supplementary information) and 
gives a typical order of magnitude for the non-intrinsic background signal originating from contact with tools, sample holders and non-clean-room environment [44].

The typical measured saturation magnetization of the Co-Sn hybrids is of the order of $10^{-8} \mathrm{Am}^{2}$. This is an order of magnitude larger than the above-mentioned non-intrinsic background, but still smaller than the saturation magnetization of the total amount of Co present in the samples. This can be understood since a fraction of Co is oxidized and, hence, does not contribute to the saturation magnetization. Furthermore, the contribution of Co which is incorporated into SnCo to the magnetization is smaller than for pure Co. Also, the magnetic hysteresis loops are not yet fully saturated, which results in an underestimation of the actual saturation magnetization.

The superconducting critical temperature $T_{C}$ of the $\mathrm{Sn}$-Co assemblies has been determined by measuring the magnetization as a function of temperature in a low magnetic field. $T_{C}$ is defined as the onset in the diamagnetic response [20]. A paramagnetic (1/T) background was subtracted from the $M(T)$ curves of the Sn-Co assemblies. The $M(T)$ curves are measured in a broad temperature range, typically up to $12 \mathrm{~K}$. Above $T_{C}$, where no diamagnetic superconducting signal is present, the measured curve is fitted with a $M(T)=A / T+B$ dependence. This fit is subtracted from the measured $M(T)$ curve in the entire temperature range, revealing only the diamagnetic superconducting response from which the superconducting transition temperature was determined. The width of the transition is $\sim 1 \mathrm{~K}$ for the pure Sn film, and amounts up to $2.5 \mathrm{~K}$ for the Sn-Co hybrid systems due to the heterogeneous composition and morphology. By measuring the critical temperature at different fixed applied magnetic fields, the superconducting phase boundary can be constructed. Figure 7 shows the $\left(H_{\text {applied }}, T\right)$-phase boundary for the Sn film and Sn-Co systems with Co concentrations of $14 \%$ and $24 \%$. 


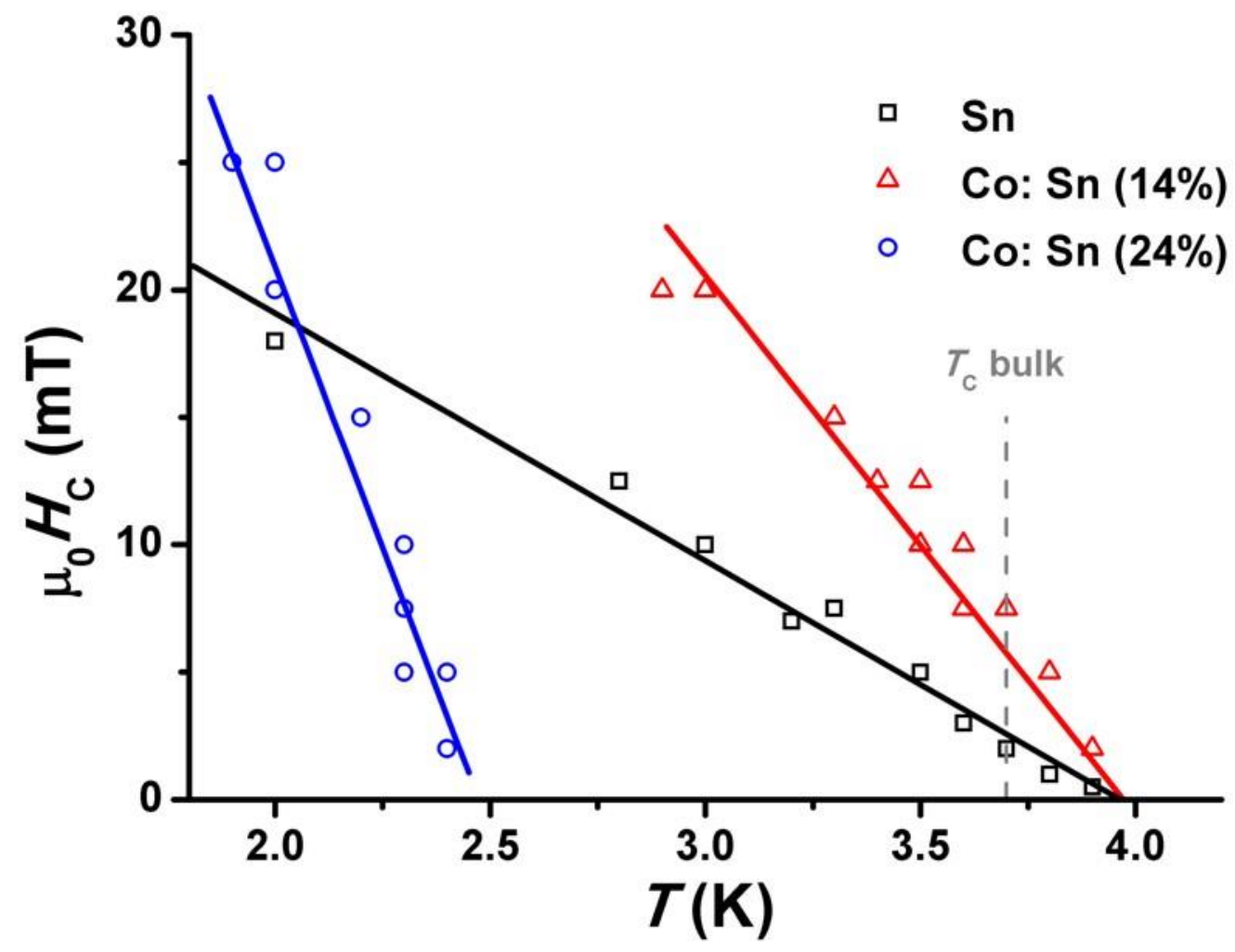

Figure 7. Superconducting phase boundaries obtained from SQUID magnetometry for a Sn film and Sn-Co hybrid systems with a Co concentration of $14 \%$ and $24 \%$.

A $T_{C}$ of $3.9 \mathrm{~K}$ is obtained for the $\mathrm{Sn}$ film which is slightly higher than $T_{C}(=3.72 \mathrm{~K})$ of the corresponding bulk material. This increase in $T_{C}$ can be attributed to the island-like and polycrystalline structure of the Sn film. A similar enhancement of $T_{C}$ has also been observed in other systems with granular nature, such as quench-condensed Sn films [45], multilayers of Al and Sn [46], and slightly oxidized Sn films [47], and is attributed to phonon confinement effects.

The phase boundary of the Sn-Co system with $14 \%$ Co shows no change in $T_{C}$ compared to the pure Sn film. For a Co concentration of $24 \%$, superconductivity is still observed with a $T_{C}$ of $2.4 \mathrm{~K}$. Only for the highest Co concentration (44\% Co), the superconducting transition (if any) has dropped below $1.8 \mathrm{~K}$ and could not be observed within our experimental means. It seems surprising that such high Co concentrations of $24 \%$ and more can still render superconducting nanocomposites. The $T_{C}$ of these Sn-Co hybrid systems is influenced by a combination of different effects. Phonon confinement effects due to the granular nature of the Sn-Co systems also occur in this case and may cause $T_{C}$ to increase to 
some extent [45 - 47]. On the other hand, phenomena like the proximity effect and the magnetic pair breaking effect will result in a decrease of $T_{C}$. The relative strength of the different effects that influence $T_{C}$ are determined by the details of the complex morphology and the interfaces between the superconducting, non-superconducting, and magnetic components in the hybrid system. Despite the very large interface area between superconducting and magnetic parts that is naturally created in hybrid nanocomposites with nanometer scale constituents, the intimate electronic interactions (e.g. through the proximity effect) are strongly suppressed if the interface transparency is low. The granular morphology of the Sn-Co hybrids (see figures 1 and 2) results in many disordered grain boundaries and a non-optimal contact area between superconducting and magnetic grains. Moreover, the formation of Sn-Co compounds (figures 3 and 5) at the S/F interfaces further lowers the interface transparency in these systems. This low interface transparency will reduce the proximity effect as one of the main mechanisms to suppress the transition temperature. We can therefore attribute the coexistence of superconductivity and ferromagnetism at high Co concentrations to the low interface transparency which in turn is a direct consequence of the specific morphology and heterogeneous composition of these nanocomposite hybrids.

As can be seen from figure 7, the phase boundaries of all samples show a linear behaviour $H_{C 2}=$ $H_{C 2}(0)\left(1-T / T_{C}\right)$ around $\mathrm{T} \approx T_{C}$, consistent with the behaviour of dirty type II superconductors (with mean free path $l<<\xi_{0}$ ). The slope of the phase boundary is found to increase for increasing Co concentration. This is attributed to a reduction of the mean free path $l$ (i.e. increase of scattering and disorder) as the Co concentration increases, leading to a smaller coherence length [48] $\left(\xi(0)=0.855 \sqrt{\xi_{0} l}\right)$ and a higher critical field $[48]\left(H_{C 2}(0)=\Phi_{0} / 2 \pi \xi(0)^{2}\right)$. Here, $\xi(T)$ is the temperature-dependent coherence length, $\phi_{0}$ is the magnetic flux quantum $\left(2.067833758 \times 10^{-15} \mathrm{~Wb}\right)$ and $\xi_{0}$ is the temperature-independent coherence length (material dependent, $\xi_{0}=230 \mathrm{~nm}$ for Sn [49]). The upper critical field can be estimated by a linear extrapolation of the superconducting phase boundary to zero Kelvin.

The values of the critical temperature, critical magnetic field, coherence length and mean free path extracted from the phase boundary of each sample are listed in table 2. From this table, it can be 
observed that $H_{C 2}$ can be substantially increased by increasing the Co content, without too much decrease in $T_{C}$.

Table 2. Sample characteristics (critical temperature $\left(T_{C}\right)$, critical magnetic field $\left(H_{C 2}\right)$, coherence length $(\xi(0))$ and mean free path $(l))$ extracted from the superconducting phase boundaries.

\begin{tabular}{ccccc}
\hline $\begin{array}{c}\text { Sample }(\text { vol. } \% \\
\text { Co })\end{array}$ & $\mathrm{T}_{\mathrm{C}}(\mathrm{K})$ & $\mu_{0} \mathrm{H}_{\mathrm{C} 2}(\mathrm{mT})$ & $\xi(0)(\mathrm{nm})$ & $l(\mathrm{~nm})$ \\
\hline Bulk Sn & 3.72 & 30.5 & - & - \\
0 & $3.9 \pm 0.1$ & $39 \pm 4$ & $93 \pm 5$ & $52 \pm 5$ \\
$14 \pm 3$ & $3.9 \pm 0.1$ & $59 \pm 4$ & $80 \pm 5$ & $39 \pm 4$ \\
$24 \pm 4$ & $2.4 \pm 0.1$ & $109 \pm 10$ & $55 \pm 3$ & $18 \pm 2$ \\
$44 \pm 5$ & - & - & - & - \\
\hline
\end{tabular}

Figure 8 shows the $M\left(H_{\text {applied }}\right)$ hysteresis curves measured at low temperature for pure $\mathrm{Sn}$ and Sn-Co hybrids with $14 \%, 24 \%$ and $44 \%$ Co concentration. The observed magnetic and superconducting behaviour in the different samples can be explained by the morphology proposed in figure 2 .

The $M\left(H_{\text {applied }}\right)$ curve of the pure Sn film (figure 8 (a)) is strongly hysteretic and flux jumps, also called flux avalanches [50], can be observed at lower fields where the magnetization becomes very large and appears noisy with sudden jumps. Both, the large hysteresis and the avalanches, indicate that strong flux pinning occurs within the superconducting Sn film, where the island boundaries in the island-like morphology of the pure Sn film serve as strong pinning sites for the flux lines. The occurrence of flux avalanches in strong pinning samples can be understood within the Bean critical state model [51]: a large constant flux gradient across a sample is stabilized due to the equilibrium between the strong pinning force and the external magnetic pressure. Fluctuations in temperature can locally help to overcome the pinning force on the flux lines, enabling movement of flux lines. Flux line motion causes dissipation which will locally increase temperature. This will promote depinning of even more flux lines, eventually resulting in flux avalanches [50, 52].

The $M\left(H_{\text {applied }}\right)$ curves of the Sn-Co hybrids with $14 \%$ Co (figure $\left.8(\mathrm{~b})\right)$ and $24 \%$ Co (figure $8(\mathrm{c})$ ) are combinations of ferromagnetic and superconducting loops. Above $\sim 30 \mathrm{mT}$, the magnetic response is dominated by the ferromagnetic behaviour. Below $\sim 30 \mathrm{mT}$, ferromagnetism and superconductivity coexist within the system. Surprisingly, while the $14 \%$ Sn-Co hybrid displays a reversible superconducting loop, the $24 \%$ Sn-Co hybrid displays a strongly irreversible superconducting loop. The very low magnetization signal and reversible $M\left(H_{\text {applied }}\right)$ behaviour for the $14 \%$ Sn-Co system 
(figure 8(b)) indicate the absence of or limited flux pinning in this system and are consistent with the behaviour of individual decoupled superconducting islands in which Meissner currents can flow [20]. The behaviour of the $M\left(H_{\text {applied }}\right)$ curve is consistent with the morphology obtained from AFM (figure 1 (b)) and proposed in figure 2 (b), showing this hybrid consists of decoupled Sn islands with reduced size.

A strong hysteresis in the superconducting response is observed for the $24 \%$ Sn-Co hybrid (figure 8(c)), indicating the presence of large Meissner currents and strong vortex pinning. This different behaviour is attributed to a change in the morphology of the $24 \%$ Sn-Co hybrid. According to the morphology proposed in figure 2(c), Sn forms small areas connecting into a percolating superconducting path, allowing Meissner currents to flow throughout the entire system. This morphology allows pinning of flux lines, consistent with the irreversible superconducting response. It should be noted that flux pinning in this Sn-Co hybrid is less strong than for the pure Sn film. Contrary to the pure Sn film, no flux avalanches were observed in this system.

Figure $8(\mathrm{~d})$ shows the hysteresis loop of the $44 \%$ Co sample at $1.8 \mathrm{~K}$. The signal stems from the ferromagnetic constituents in the samples, no superconducting contribution could be observed down to $1.8 \mathrm{~K}$, the lowest temperature that can be reached in our SQUID magnetometer. Note that the ferromagnetic response for this sample at low temperature is only slightly higher than at room temperature (figures 8(d) and 6(c)). Also for the other samples, we can expect that the magnetic signal is not strongly temperature dependent in this temperature range (between 2 and $300 \mathrm{~K}$ ). While there is no macroscopic superconductivity (above $1.8 \mathrm{~K}$ ) observed for the sample with $44 \% \mathrm{Co}$, it can not be excluded that there is local superconductivity within the isolated Sn areas. 
(a)
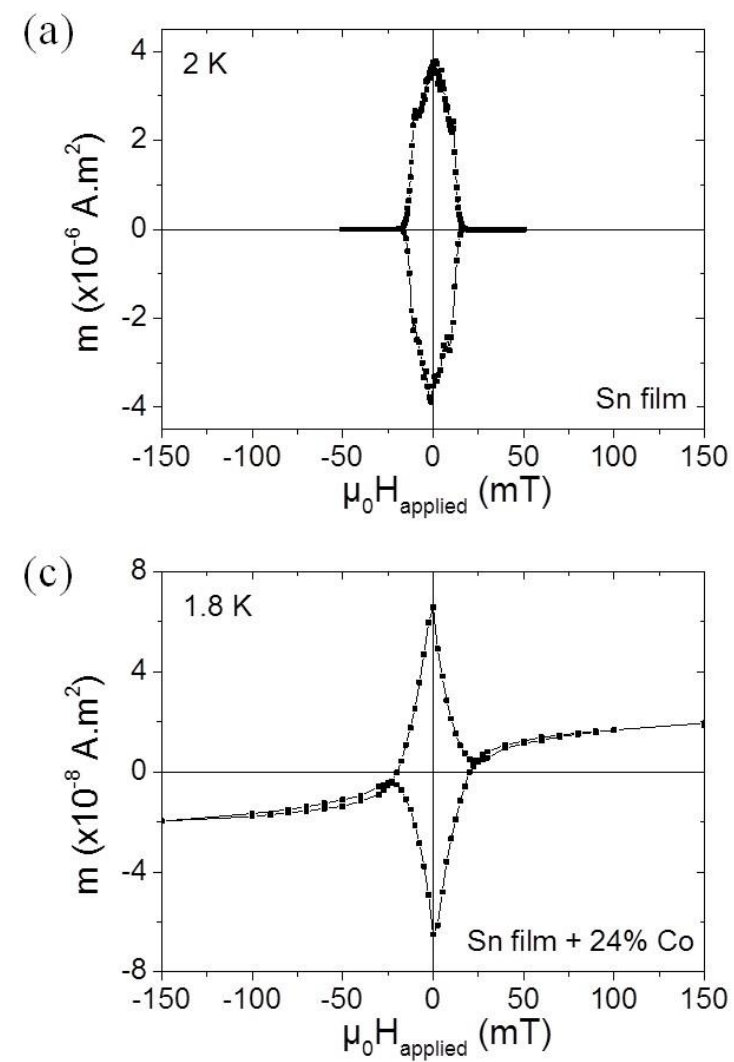
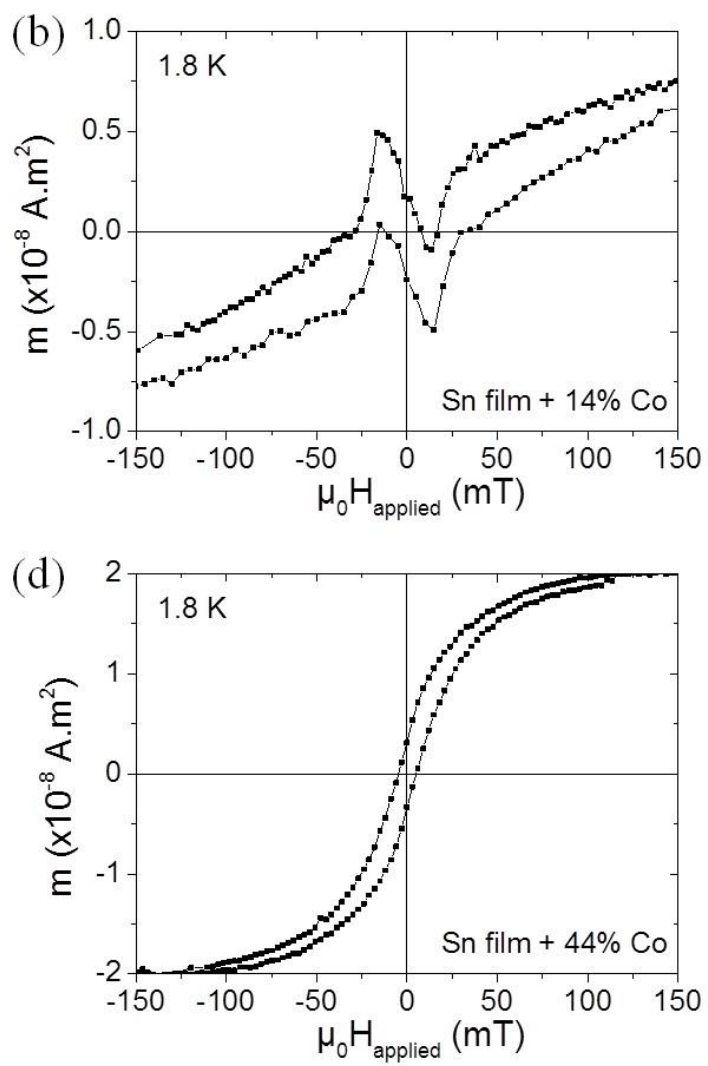

Figure 8. Magnetic moment as a function of the applied magnetic field at low temperatures for Sn-Co systems with different Co concentrations; (a): pure Sn film; (b): Sn film with 14\% Co; (c): Sn film with $24 \%$ Co; (d): Sn film with $44 \%$ Co.

\section{Conclusions}

S/F Sn-Co hybrid systems with Co concentrations ranging up to 44 vol.\% have been created by codepositing preformed gas phase Co clusters and thermally evaporated Sn atoms. The morphology is determined by the interplay of diffusion and coalescence of the Co clusters and formation of different Sn-Co compounds. By tuning the deposition rate of the Co clusters relative to that of the $\mathrm{Sn}$ atoms, the morphology as well as the composition of the S/F nanocomposites can be manipulated. Based on detailed complementary (GI)XRD, AFM and CEMS characterization, a growth model has been presented showing that the morphology evolves from flat $\mathrm{Sn}$ islands to granular nanocomposites with increasing Co cluster flux. Superconducting and magnetic properties for increasing Co content indicate that all Co-containing hybrids are ferromagnetic and, except for the highest Co-concentration (44\% Co), also remain superconducting with increasing critical field and decreasing $T_{C}$ as the Co content increases. The fact that superconductivity can persist up to high Co concentrations (24\% Co 
and more) is attributed to the specific nanogranular morphology and the partial mixing of Sn and Co at the $\mathrm{Sn} / \mathrm{Co}$ interfaces which result in a low electronic interface transparency between $\mathrm{Sn}$ and Co. The Meissner response was shown to vary from a reversible to a strongly hysteretic behaviour depending on the morphology of the samples with different Co content. This was explained in terms of the strong dependence of flux pinning on the morphological features with length scales comparable to the penetration depth and coherence length. It is exactly at these length scales that the morphology is affected by varying the Co content.

This study illustrates the intimate interplay between morphology, composition, superconductivity and magnetism in complex nanocomposites. Nanoscale morphology and composition can have in particular a strong influence on the hybrid interfaces and on the electronic coupling between the $\mathrm{S}$ and F constituents. While this does not influence as much the magnetic pair breaking (e.g. through stray fields), it does strongly reduce the electronic proximity effect as pair breaking mechanism. This results in a coexistence of $\mathrm{S}$ and $\mathrm{F}$ up to high $\mathrm{F}$ content in systems where $\mathrm{S}$ and $\mathrm{F}$ interact mainly via magnetic fields.

\section{Acknowledgements}

The authors would like to thank P. Lievens for fruitful discussions and C. Van Haesendonck for use of SPM equipment. This work was supported by the Research Foundation-Flanders (FWO), the Concerted Research Action (GOA 14/007) and SPIRIT (Support of Public and Industrial Research using Ion beam Technology, contract No. 227012). K.H., E.M. and T.P. thank the FWO for financial support.

\section{References}

[1] A. I. Buzdin, Proximity effects in superconductor-ferromagnet heterostructures, Rev. Mod. Phys. 77 (2005) 935.

[2] M. J. Van Bael, K. Temst, V. V. Moshchalkov and Y. Bruynseraede, Magnetic properties of submicron Co islands and their use as artificial pinning centers, Phys. Rev. B 59 (1999) 14674.

[3] J. I. Martin, M. Vélez, J. Nogues and I. K. Schuller, Flux pinning in a superconductor by an array of submicrometer magnetic dots, Phys. Rev. Lett. 79 (1977) 1929.

[4] Y. Jaccard, J. I. Martin, M. C. Cyrille, M. Vélez, J. L. Vicent and I. K. Schuller, Magnetic pinning of the vortex lattice by arrays of submicrometric dots, Phys. Rev. B 58 (1998) 8232. 
[5] M. J. Van Bael, J. Bekaert, K. Temst, L. Van Look, V. V. Moshchalkov, Y. Bruynseraede, G. D. Howells, A. N. Grigorenko, S. J. Bending and G. Borghs, Local observation of field polarity dependent flux pinning by magnetic dipoles, Phys. Rev. Lett. 86 (2001) 155.

[6] M. Lange, M. J. Van Bael, Y. Bruynseraede and V. V. Moshchalkov, Nanoengineered magneticfield-induced superconductivity, Phys. Rev. Lett. 90 (2003) 197006.

[7] D. Stamopoulos, M. Pissas, V. Karanasos, D. Niarchos and I. Panagiotopoulos, Influence of randomly distributed magnetic nanoparticles on surface superconductivityin Nb films, Phys. Rev. B 70 (2004) 054512.

[8] Z. Yang, M. Lange, A. Volodin, R. Szymczak and V. V. Moshchalkov, Domain-wall superconductivity in superconductor-ferromagnet hybrids, Nature Materials 3 (2004) 793-798. [9] B. A. Young, J. R. Williams, S. W. Deiker, S. T. Ruggiero and B. Cabrera, Using ion implantation to adjust the transition temperature of superconducting films, Nuclear Instruments and Methods in Physics Research Section A: Accelerators, Spectrometers, Detectors and Associated Equipment 520 (2004) 307-310.

[10] P. Jalkanen, V. Tuboltsev, A. Virtanen, K. Arutyunov, J. Räisänen, O. Lebedev and G. Van Tendeloo, Critical temperature modification of low dimensional superconductors by spin doping, Solid State Commun. 142 (2007) 407-411.

[11] J. E. Crow, R. P. Guertin and R. D. Parks, Superconducting and magnetic properties of dilute paramagnetic metals, Phys. Rev. Lett. 19 (1967) $77-81$.

[12] K. H. Bennemann, Anomalous Dependence of the Superconducting Transition Temperature on Paramagnetic Impurities, Phys. Rev. Lett. 17 (1966) 438 - 443.

[13] J. J. Hauser, M. Robbins and F. J. DiSalvo, Effect of 3 d Impurities on the Superconducting Transition Temperature of the Layered Compound $\mathrm{NbSe}_{2}$, Physical Review B 8 (1973) 1038 - 1042.

[14] M. Iavarone, G. Karapetrov, J. Fedor, D. Rosenmann, T. Nishizaki and N. Kobayashi, The local effect of magnetic impurities on superconductivity in $\mathrm{Co}_{x} \mathrm{NbSe}_{2}$ and $\mathrm{Mn}_{\mathrm{x}} \mathrm{NbSe}_{2}$ single crystals, Journal of Physics: Condensed Matter 22 (2010) 015501.

[15] Y. T. Xing, H. Micklitz, T. G. Rappoport, M. V. Milošević, I. G. Solórzano-Naranjo and E. Baggio-Saitovitch, Spontaneous vortex phases in superconductor-ferromagnet $\mathrm{Pb}$-Co nanocomposite films, Phys. Rev. B 78 (2008) 224524.

[16] Y. T. Xing, H. Micklitz, E. Baggio-Saitovitch and T. G. Rappoport, Controlled switching between paramagnetic and diamagnetic Meissner effects in superconductor-ferromagnet $\mathrm{Pb}-\mathrm{Co}$ nanocomposites, Phys. Rev. B 80 (2009) 224505.

[17] J. Aarts, J. M. E. Geers, E. Brück, A. A. Golubov and R. Coehoorn, Interface transparency of superconductor/ferromagnetic multilayers, Phys. Rev. B 56 (1997) 2779.

[18] M. G. Flokstra, T. C. Cunningham, J. Kim, N. Satchell, C. H. Marrows, G. Burnell, S. J. Bending, P. J. Curran, S. J. Langridge, C. Kinane, J. F. K. Cooper, N. Pugach, M. Eschrig and S. L. Lee, Controlled suppression of superconductivity by the generation of polarized Cooper pairs in spin valve structures, arXiv Preprint 1404.2950v2 (2014).

[19] N. Banerjee, C. B. Smiet, R. G. J. Smits, A. Ozaeta, F. S. Bergeret, M. G. Blamire and J. W. A. Robinson, Evidence for spin selectivity of triplet pairs in superconducting spin valves, Nature Communications 5 (2014) 3048.

[20] J. Cuppens, C. P. Romero, P. Lievens and M. J. Van Bael, Superconductivity in Pb cluster assembled systems with different degrees of coagulation, Phys. Rev. B 81 (2010) 064517.

[21] A. Perez, P. Melinon, V. Dupuis, P. Jensen, B. Prevel, J. Tuaillon, L. Bardotti, C. Martet, M.

Treilleux, M. Broyer, M. Pellarin, J. L. Vaille, B. Palpant and J. Lerme, Cluster assembled materials: a novel class of nanostructured solids with original structures and properties, J. Phys. D: Appl. Phys. 30 (1997) $709-721$.

[22] J. Stahn, J. Chakhalian, C. Niedermayer, J. Hoppler, T. Gutberlet, J. Voigt, F. Treubel, H. Habermeier, G. Cristiani, B. Keimer and C. Bernhard, Magnetic proximity effect in perovskite superconductor/ferromagnet multilayers, Phys. Rev. B 71 (2005) 140509.

[23] J. Chakhalian, J. W. Freeland, G. Srajer, J. Strempfer, G. Khaliullin, J. C. Cezar, T. Charlton, R. Dalgliesh, C. Bernhard, G. Cristiani, H. Habermeier and B. Keimer, Magnetism at the interface between ferromagnetic and superconducting oxides, Nature Physics 2 (2006) 244.

[24] Y. Sun, M. B. Salamon, K. Garnier and R. S. Averback, Glassy vortex dynamics induced by a random array of magnetic particles above a superconductor, Phys. Rev. Lett. 92 (2004) 097002. 
[25] A. Palau, H. Parvaneh, N. A. Stelmashenko, H. Wang, J. L. Macmanus-Driscoll and M. G. Blamire, Hysteretic vortex pinning in superconductor-ferromagnet nanocomposites, Phys. Rev. Lett. 98 (2007) 117003-117003.

[26] N. W. Ashcroft and N. D. Mermin, Solid State Physics, Philadelphia : Saunders College, 1976.

[27] N. Vandamme, E. Janssens, F. Vanhoutte, P. Lievens and C. Van Haesendonck, Scanning probe microscopy investigation of gold clusters deposited on atomically flat substrates, J. Phys.: Condens. Matter 15 (2003) 2983 - 2999.

[28] R. A. Young, The Rietveld Method, Vol. 1, Oxford: Oxford University Press, 1995.

[29] MAUD (Materials Analysis Using Diffraction), http://www.ing.unitn.it/ maud. Access date: 02/05/2014.

[30] U. Gonser, Mössbauer Spectroscopy, Berlin: Springer-Verlag, 1975.

[31] D. G. Rancourt and J. Y. Ping, Voigt-based methods for arbitrary-shape static hyperfine parameter distributions in Mössbauer spectroscopy, Nuclear Instruments and Methods in Physics

Research Section B: Beam Interactions with Materials and Atoms 58 (1991) 85-97.

[32] J. G. Stevens, Isomer shift reference scales, Hyperfine Interactions 13 (1983) 221-236.

[33] V. N. Popok, Energetic cluster ion beams: Modification of surfaces and shallow layers, Materials Science and Engineering: R: Reports 72 (2011) 137-157.

[34] C. Binns, Nanoclusters deposited on surfaces, Surf. Sc. Rep. 44 (2001) 1-49.

[35] I. Petrov, P. B. Barna, L. Hultman and J. E. Greene, Microstructural evolution during film growth, J. Vac. Sci. Technol. A 21 (2003) 117-128.

[36] S. Hishita, Z. Stryhal, I. Sakaguchi, N. Ohashi, N. Saito and H. Haneda, Sn film deposition on silica glass substrates, Thin Solid Films 464 (2004) 146-149.

[37] H. Luo and P. Duwez, Face-centered Cubic Cobalt-rich Solid Solutions in Binary Alloys with Aluminum, Gallium, Silicon, Germanium, and Tin, Canadian Journal of Physics 41 (1963) 758-761. [38] K. H. J. Buschow, P. G. Van Engen and R. Jongebreur, Magneto-optical properties of metallic ferromagnetic materials, J. Magn. Magn. Mater. 38 (1983) 1-22.

[39] S. Stankov, B. Sepiol, T. Kanuch, D. Scherjau, R. Würschum and M. Miglierini, High temperature Mössbauer effect study of $\mathrm{Fe}_{90} \mathrm{Zr}_{7} \mathrm{~B}_{3}$ nanocrystalline alloy, J. Phys.: Condens. Matter 17 (2005) 3183.

[40] R. Alcantara, I. Rodriguez and J. L. Tirado, Structural and Electrochemical Properties of Micro-and Nano-Crystalline CoSn Electrode Materials, Chem. Phys. Chem. 9 (2009) 1171-1177.

[41] J. Jaén, M. L. Varsányi, E. Kovács, I. Czakó-Nagy, A. Buzás, A. Vértes and L. Kiss, Structural studies of electrodeposited tin — cobalt alloys, Electrochimica Acta 29 (1984) 1119-1122.

[42] K. H. J. Buschow and F. R. De Boer, Physics of Magnetism and Magnetic Materials, Vol. 92, Kluwer Academic/Plenum Publishers, 2003.

[43] G. Marchal, D. Teirlinck, P. Mangin, C. Janot and J. Hübsch, Magnetic Behaviour of Vapour Quenched $\mathrm{Co}_{\mathrm{x}} \mathrm{Sn}_{1-\mathrm{x}}$ Alloys, Le Journal de Physique Colloques 41 (1980) C8-662.

[44] L. M. C. Pereira, J. P. Araujo, M. J. Van Bael, K. Temst and A. Vantomme, Practical limits for detection of ferromagnetism using highly sensitive magnetometry techniques, J. Phys. D: Appl. Phys. 44 (2011) 215001.

[45] A. Shalnikov, Superconducting thin films, Nature 142 (1938) 74.

[46] B. Abeles, R. W. Cohen and G. W. Cullen, Enhancement of superconductivity in metal films, Phys. Rev. Lett. 17 (1966) 632.

[47] M. Strongin, O. F. Kammerer, J. E. Crow, R. D. Parks, J. D. H. Douglass and M. A. Jensen, Enhanced superconductivity in layered metallic films, Phys. Rev. Lett. 21 (1968) 1320.

[48] M. Tinkham, Introduction to Superconductivity, Courier Dover Publications, 1975.

[49] C. Kittel and P. McEuen, Introduction to Solid State Physics, Vol. 8, New York: Wiley, 1976.

[50] E. Altshuler and T. H. Johansen, Colloquium: Experiments in vortex avalanches, Rev. Mod. Phys. 76 (2004) 471.

[51] C. P. Bean, Magnetization of hard superconductors, Phys. Rev. Lett. 8 (1962) 250.

[52] S. Hébert, L. Van Look, L. Weckhuysen and V. V. Moshchalkov, Vortex avalanches in a Pb film with a square antidot array, Phys. Rev. B 67 (2003) 224510. 